\title{
A Low-Voltage Low-Power Fully-Integratable Automatic Gain Control for Hearing Instruments
}

\author{
Wouter A. Serdijn, Albert C. van der Woerd, Jan Davidse, and Arthur H. M. van Roermund
}

\begin{abstract}
A low-voltage low-power bipolar automatic gain control (AGC) for hearing instruments that works in the current domain and operates on a single 1.3-V battery is presented. In this AGC a large time constant $(50 \mathrm{~ms})$ is realized on-chip. The AGC consists of a gain cell, a comparator and a voltage follower. The active circuitry of the AGC has been integrated in the DIMES01 process and the total circuit demonstrates operation down to 1 $\mathrm{V}$ with only $4 \mu \mathrm{W}$ power consumption. The compression range amounts to $38 \mathrm{~dB}$. The AGC has a dynamic range (DR) of $62 \mathrm{~dB}$ at the output over a bandwidth of $10 \mathrm{kHz}$.
\end{abstract}

\section{INTRODUCTION}

A PART from pitch, loudness and timbre, information in the world of sound is also characterized by more or less sudden temporary changes. For someone with hearing impairment these variations do not fit in his or her dynamic range and therefore there is either the lack of certain parts of the information or the pain limit is frequently exceeded. In this situation and automatic gain control (AGC) can offer certain improvement of the (speech) intelligibility.

An AGC is a circuit that automatically controls its gain in such a way that variations of the input signal result in smaller variations of the output signal. This control action is usually performed by means of a loop that contains a large time constant (e.g., several tens of milliseconds). In the past, this large time constant was realized by means of a large (external) capacitor [1], [2]. However, to reduce the size and production costs to a minimum, external components need to be avoided as much as possible. This also holds for the power supply; the use of more than one single zinc-air battery is precluded. This means that the AGC has to operate at "low-voltage level" (i.e., $1-1.3 \mathrm{~V}$ ) and consume as little current as possible to ensure long battery life.

In practice two kinds of AGC's are found [3]: the inputcontrolled AGC-I and the output-controlled AGC-O. The AGC-I obtains its control signal from a signal in front of the volume control. In this way the control action will depend on the sound pressure level at the input of the hearing instrument. The control signal of the AGC-O is derived from the signal behind the volume control. The control action then depends on the sound pressure level that is offered to the ear.

Both AGC's differ from each other so far as that the control range of the AGC-I is always larger (for example, $60 \mathrm{~dB}$ ) while for the AGC-O often $20 \mathrm{~dB}$ is enough. Also the compression

Manuscript received December 2, 1993; revised April 1, 1994.

The authors are with the Department of Electrical Engineering, Delft University of Technology, $2628 \mathrm{CD}$ Delft, the Netherlands.

IEEE Log Number 9402464.

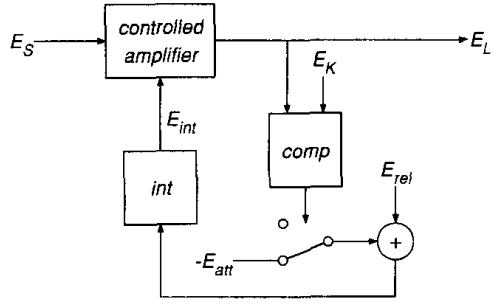

Fig. 1. Block diagram of an automatic gain control.

ratio is different: e.g., between $1.5-10$ for the AGC-I and infinite for the AGC-O.

The circuit that is described here is an AGC-O with an infinite compression ratio. Section 2 presents a block diagram of an AGC that operates in the current domain, consisting of three sub-circuits: a gain cell, a comparator, and a voltage follower. Their design is presented in Section 3. In Section 4 the circuit diagram of the complete AGC is presented. As an example the active circuitry was integrated in the DIMES01 process. In Section 5 experimental results are given.

\section{AN AGC IN THE CurRent Domain}

In Fig. 1 a typical AGC circuit is drawn. The output signal $E_{L}$ is compared with a reference level $E_{K}$ (the knee level) by a comparator that determines whether the integrating circuit-in practice often nothing more than an $R C$ network-is charged (by $E_{\mathrm{att}}-E_{\mathrm{rel}}$ ) or discharged (by $E_{\mathrm{rel}}$ ). The output signal of the integrator $E_{\text {int }}$ forms the control signal of the controlled amplifier. The operation is as follows: when $E_{\text {att }}$ is larger than $E_{\text {rel }}$ the output signal $E_{L}$ is controlled towards the knee level $E_{K}$. Variations of the input signal therefore always result in smaller or equal variations of the output signal. The control action needs some time. This can be described by the expressions attack time and release time. The attack time is defined as the time needed for the AGC to respond to a sudden $25 \mathrm{~dB}$ increase of the input signal until the output signal is within $2 \mathrm{~dB}$ from its final value [4]. Vice versa, the release time is defined as the time needed to respond to a sudden 25 $\mathrm{dB}$ decrease of the input signal until the output signal is within $2 \mathrm{~dB}$ from its final value. The attack time and the release time must be $<5 \mathrm{~ms}$ and $50 \mathrm{~ms}$, respectively.

Low-voltage low-power circuits preferably operate in the current domain [5]. This leads to the circuit diagram of Fig. 2. Apart from $E_{\text {int }}$ all signals are now represented by currents. The output of the integrator is a voltage, as the 


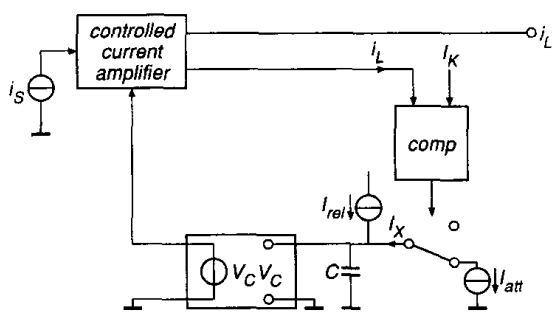

Fig. 2. Block diagram of an AGC operating in the current domain.

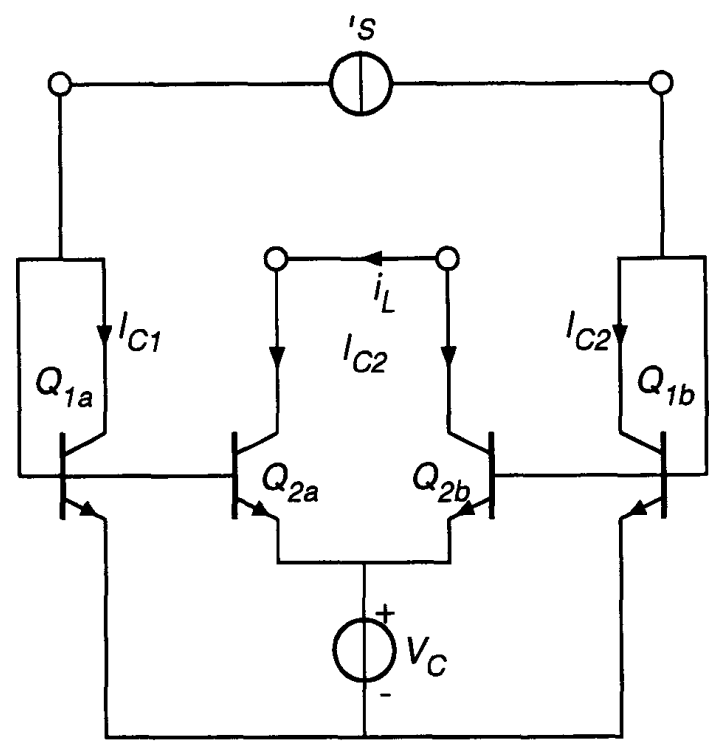

Fig. 3. A symmetrical current mirror, also known as a Beta-Immune Type 'A' Cell.

only integratable integrating element is a capacitance. The capacitance is followed by a voltage follower that generates a low-impedance version of $V_{C}$.

\section{Design Of THE SubciRCUITS}

So far the AGC has been designed at system level. We now take a closer look at the design of its components: the controlled amplifier, the comparator (including the switch and current source $I_{\text {att }}$ ) and the voltage follower.

\section{A. Design of the Controlled Amplifier}

A suitable controlled amplifier is the symmetrical version of a current mirror, also known as a Beta-Immune Type 'A' Cell [6, Chapter 2] (Fig. 3). This is a controlled current amplifier of which the gain equals the ratio of the (dc) collector currents $I_{C 2}$ and $I_{C 1}$.

Another way of controlling the ratio of $I_{C 2}$ and $I_{C 1}$ is by means of controlling voltage $V_{C}$ connected between the emitter of $Q_{1}$ and the emitter of $Q_{2}$. We now obtain a gain, $G$, that

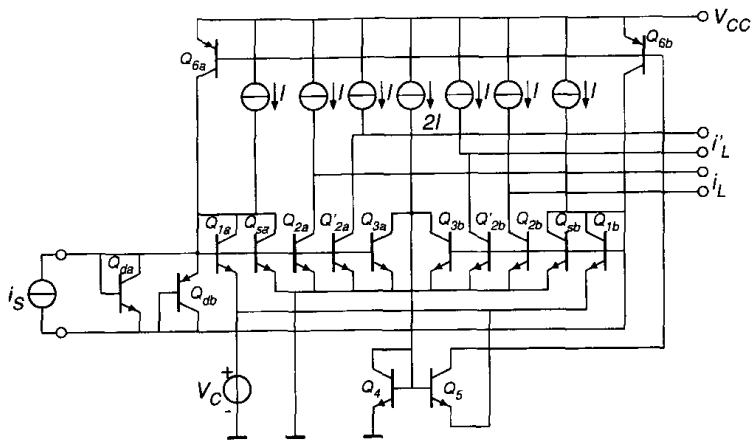

Fig. 4. A symmetrical current mirror as used in the controlled amplifier including its biasing circuitry.

is proportional to the anti-log of $V_{C}$, or

$$
G=i_{C 2} / i_{C 1}=e^{V_{C} / V_{T}} \approx 335 V_{C} \mathrm{~dB}, V_{C} \text { in } \mathrm{V}
$$

in which $V_{T}$ equals the thermal voltage $k T / q$, approximately $26 \mathrm{mV}$ at $300 \mathrm{~K}$.

This exponential relationship between the gain $G$ and the control voltage $V_{C}$ enables us to control the gain over a wide range and is also perceptibly the most comfortable. If $V_{C}$ is made proportional to the absolute temperature, the gain is independent of the temperature as well.

A possible implementation of a voltage-controlled symmetrical current mirror is given in Fig. 4. Transistors $Q_{1 a}$ and $Q_{1 b}$ are the input transistors. $Q_{2 a}$ and $Q_{2 b}$ deliver the output current $i_{L} \cdot Q_{2 a}^{\prime}$ and $Q_{2 b}^{\prime}$ are the output transistors for $i_{L}^{\prime}$. The circuit also contains a common-mode loop [7]-[9] for biasing the transistors correctly $\left(Q_{3 a}, Q_{3 b}, Q_{4}, Q_{5}, Q_{6 a}\right.$, and $\left.Q_{6 b}\right)$. The collector currents of both $Q_{3 a}$ and $Q_{3 b}$, that equal the collector currents of both $Q_{2 a}$ and $Q_{2 b}$, are added and compared with a current $2 I$. The error signal controls via $Q_{4}, Q_{5}, Q_{6 a}$, and $Q_{6 b}$ the collector currents of $Q_{1 a}$ and $Q_{1 b}$. Because the gain in this loop, the common-mode loop gain, equals the current gain factor $B_{F}$ of $Q_{6 a}$ and $Q_{6 b}$, which is much larger than one, the error signal is nullified and the symmetrical current mirror is biased correctly. $Q_{s a}$ and $Q_{s b}$ limit the maximum gain of the amplifier to one. $Q_{d a}$ and $Q_{d b}$ shunt the input and prevent the amplifier from saturating.

\section{B. Design of the Comparator}

The comparator is the subcircuit that decides whether the output current $i_{L}^{\prime}$ of the controlled amplifier is larger or smaller than the reference level $I_{K}$. For this purpose we can again use a symmetrical current mirror, now acting as an amplifier with a saturated input-output relation. Its implementation is given in Fig. 5. $Q_{3 a}, Q_{3 b}, Q_{4}, Q_{5}, Q_{6 a}, Q_{6 b}$, and $Q_{6 c}$ form the common-mode biasing circuitry. In this case the commonmode loop gain is kept sufficiently low (i.e., equals 2) to prevent instability in the comparator. The output current $I_{X}$ therefore switches between 0 and $\frac{2}{3} I$. The two diode-connected transistors $Q_{d a}$ and $Q_{d b}$ prevent the amplifier from saturating; the comparator switches faster. 


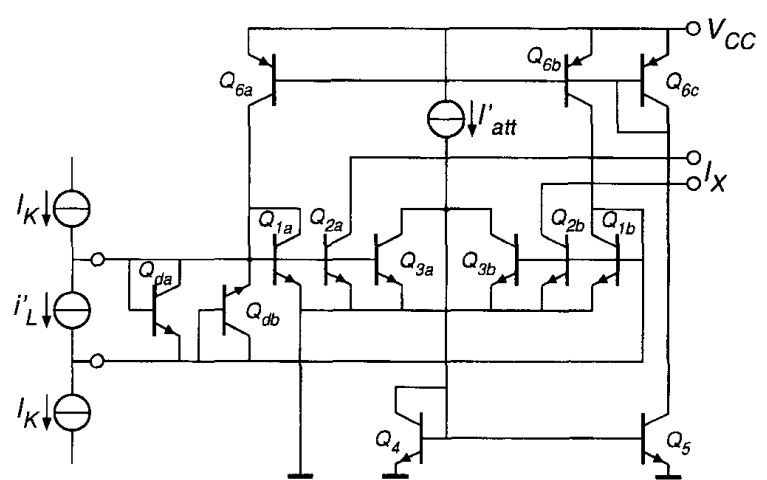

Fig. 5. A symmetrical current mirror used as a comparator. The common-mode loop gain equals two to prevent instability.

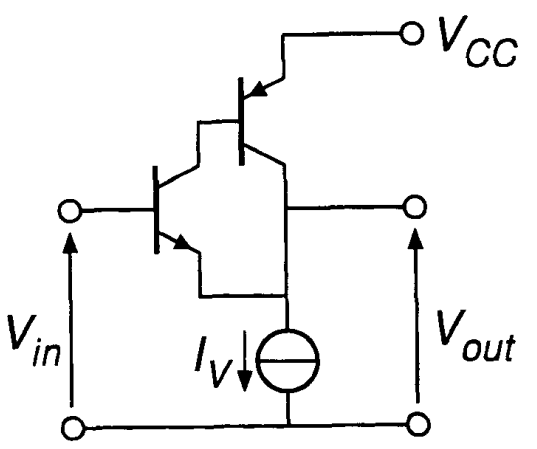

Fig. 6. A two-transistor voltage follower.

\section{Design of the Voltage Follower}

The voltage follower forms a buffer between the capacitance $C$ and the controlled amplifier. A single Field-Effect transistor (JFET or MOST) would perform this task very well, but as such devices are not yet well specified for applications in lowvoltage circuits, a sufficiently high input impedance should be created by means of negative feedback [10]. A two-transistor solution is given in Fig. 6. Although it might be confusing to see that the output voltage differs a base-emitter voltage from the input voltage, we have to consider that the voltage follower is part of a loop; the loop will control correctly.

\section{OVERALL DESIGN}

Now that all the different parts of the AGC have been designed at circuit level, all the subcircuits are linked together, and we take a closer look at the numerical values and the remaining bias circuitry.

When driving the filter as presented in [11] the maximum output current of the AGC circuit is chosen to be $25 \mathrm{nA}$ (peak value). The reference current $I_{K}$ (Fig. 2) therefore has been chosen to be equal to $25 \mathrm{nA}$. The current source $I$ as depicted

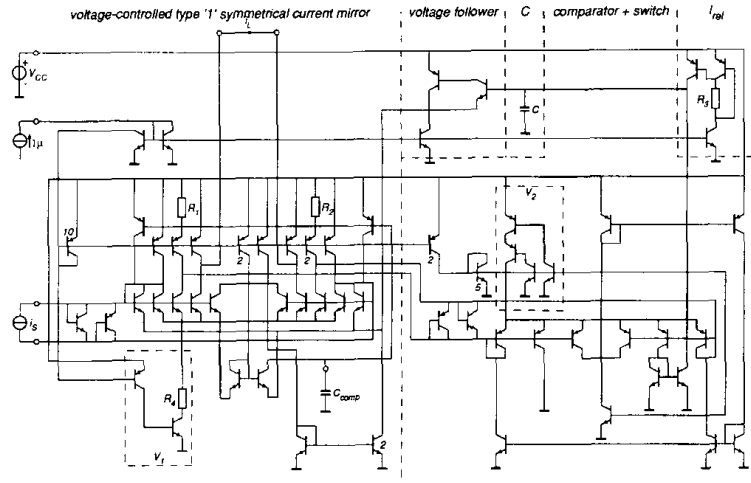

Fig. 7. The total automatic gain control. Instability may be counteracted by Comp.

in Fig. 4 has been chosen well above $25 \mathrm{nA}$ and equals 100 nA.

The values of $I_{\text {att }}$ and $I_{\text {rel }}$ can be derived from the attack time and the release time. Some calculation yields

$$
I_{\text {att }}=\frac{5.2 V_{T} C}{t_{\text {att }}}+I_{\text {rel }}
$$

and

$$
I_{\mathrm{rel}}=\frac{2.6 V_{T} C}{t_{\mathrm{rel}}} .
$$

For $I_{a t t^{\prime}}$ (Fig. 5) it follows

$$
I_{\mathrm{att}^{\prime}}=\frac{3}{2} I_{\mathrm{att}}=\frac{7.8 V_{T} C}{t_{\mathrm{att}}}+\frac{3}{2} I_{\mathrm{rel}} .
$$

With $t_{\text {att }}, t_{\text {rel }}$ and $C$ equal to $4 \mathrm{~ms}, 50 \mathrm{~ms}$, and $400 \mathrm{pF}$, respectively, this results in $20 \mathrm{nA}$ and $540 \mathrm{pA}$ for $I_{\mathrm{att}}$ and $I_{\text {rel }}$.

The current source $I_{V}$ (Fig. 6) supplies the collector current of the p-n-p transistor in the voltage follower and is chosen to be equal to $1 \mu \mathrm{A}$.

All these currents can be derived from a single current by means of current mirrors with multiple outputs and convenient scaling factors. The scaling factor can be obtained by choosing either a proper emitter area ratio or by means of resistors. The latter solution yields either a Widlar mirror or a $\mathrm{gm}$ compensated mirror [6, Chapter 6].

The total circuit diagram of the AGC is depicted in Fig. 7. Two voltage sources $\left(V_{1}\right.$ and $\left.V_{2}\right)$ have been added to prevent the current sources $I_{V}$ and $I_{K}$ from saturating. $V_{1}$ has been realized by means of a saturating $n-p-n$ transistor and a resistor. $V_{2}$ contains two saturating $\mathrm{p}-\mathrm{n}-\mathrm{p}$ transistors in series. In this way their voltages are well above the saturation voltages of $I_{V}$ and $I_{K}$.

In case of (common-mode) instability a capacitance $C_{\text {comp }}$ can be added.

\section{EXPERIMENTAL RESULTS}

The active circuitry of the circuit shown in Fig. 7 was integrated in the DIMESO1 process $(5 \mathrm{GHz}, 2 \mu \mathrm{m})$, fabricated at the Delft Institute of Microelectronics and Submicron 
TABLE I

Measurement Results of the AGC

\begin{tabular}{lll}
\hline Parameter & Value & Unit \\
Compression range & 38 & $\mathrm{~dB}$ \\
Attack time, $i_{s}=1 \mu \mathrm{A}_{p}, 1 \mathrm{kHz}$ & 4.2 & $\mathrm{~ms}$ \\
Release time, $i_{s}=10 \mathrm{nA}_{p}, 1 \mathrm{kHz}$ & 58 & $\mathrm{~ms}$ \\
Dynamic Range, $G=1 . B=10 \mathrm{kHz}$ & 62 & $\mathrm{~dB}$ \\
Bandwidth & $>100$ & $\mathrm{kHz}$ \\
Min. supply voltage & 1 & $\mathrm{~V}$ \\
Supply current, $G=1$ & 4 & $\mu \mathrm{A}$ \\
\hline
\end{tabular}

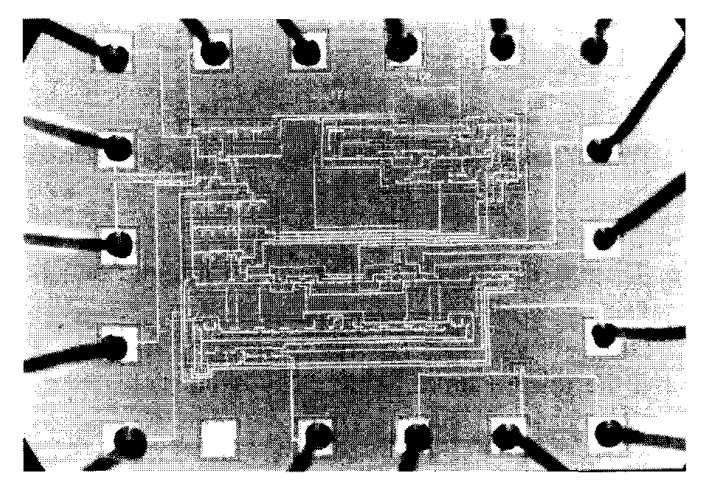

Fig. 8. Photograph of the integrated circuit.

Technology. Fig. 8 shows a microphotograph of the chip. Experiments proved the correct operation of the AGC. Table I gives the measurement results. No instability occurred. The relatively large value of the release time is caused by the base current of the first stage of the voltage follower. However, this did not pose a problem in our application.

\section{REFERENCES}

[1] F. Callias, F. H. Salchli, and D. Girard: "A set of four IC's in CMOS technology for a programmable hearing aid," IEEE J. Solid-State Circuits, vol. 24, no. 2, April 1989, pp. 301-312.

[2] M. Mauthe, U. Kleine, P. Linnert, and G. H. Steeger, "A micropower CMOS circuit for hearing aids," in Proc. ESSCIRC, Grenoble, 1990, pp. $45-48$.

[3] V. J. Geers, F. Keller, A. Löwe, and P. Plath, "Technishe Hilfe bei der Rehabilitation Hörgeschädigter," (in German) Heidelberg: SpringerVerlag, 1980

[4] I. E. C. Recommendation 118-2, Hearing aids with automatic gain control circuits, 1983

[5] W. A. Serdijn, "The design of low-voltage low-power analog integrated circuits and their applications in hearing instruments," Ph.D. thesis, Delft University of Technology, Delft, the Netherlands, 1994.

[6] C. Toumazou, F. J. Lidgey, and D. G. Haigh, Eds. Analogue IC Design: The Current-Mode Approach, London: Peter Peregrinus Ltd., 1990,

[7] P. T. M. van Zeijl, "Fundamental aspects and design of an FM upconversion receiver front-end with on-chip SAW filters," $\mathrm{Ph} . \mathrm{D}$. thesis, Delft University of Technology, Delft, the Netherlands, 1990, Ch. 5.

[8] J. van der Plas, "Synchronous detection in monolithically integrated AM upconversion receivers," Ph.D. thesis, Delft University of Technology, Delft, the Netherlands, 1990, Ch. 4.

[9] A. Payne, C. Toumazou, and P. Ryan, "Differential current input cell with common mode feedback," Electron. Lett., vol. 26, no. 20, Sept. $27,1990$.

[10] E. H. Nordholt, Design of High Performance Negative Feedback Amplifiers, Amsterdam: Elsevier Scientific Publishing Company, 1983.

[11] W. A. Serdijn, "A low-voltage low-power current-mode high-pass leapfrog filter," Analog Integrated Circuits and Signal Processing, vol. 3, pp. $105-112,1993$.

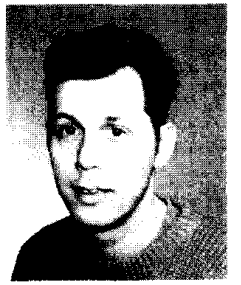

Wouter Serdijn was born in Zoetermeer, the Netherlands, in 1966. He started his study at the Faculty of Electrical Engineering at the Delft University of Technology in 1984, and received the "ingenieurs" (M.Sc.) degree in 1989.

Subsequently, he joined the Electronics Research Laboratory of the same university where he received the Ph.D. degree in 1994. His research includes developing a formal design theory for low-voltage low-power analog integrated circuits along with the development of circuits for hearing instruments.

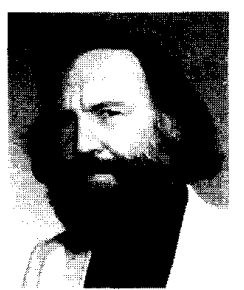

Albert C. van der Woerd was born in 1937 in Leiden, the Netherlands. In 1977 he received the "ingenieurs" (M.Sc.) degree in electrical engineering from the Delft University of Technology, Delft, the Netherlands. He was awarded the Ph.D. degree in 1985 .

From 1959 to 1966 he was engaged in research on and development of radar and TV circuits with several industry laboratories. In 1966 he joined the Electronics Research Laboratory of the Faculty of Electrical Engineering of the Delft University of Technology. During the first 11 years he carried out research on electronic musical instruments. The next 8 years his main research subject was on carrier domain devices. The last years he has been specialized in the field of low-voltage low-power analog circuits and systems. He teaches design methodology.

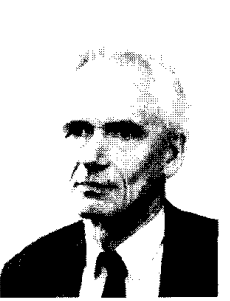

Jan Davidse received the M.Sc. degree in electrical engineering from Delft University of Technology in 1953 and subsequently the Ph.D. degree from Eindhoven University of Technology.

He was research scientist with Philips Research Laboratories and received a full professorship in electronics at the Delft University of Technology in 1964. From 1964 to the present, he has been head of the laboratory for Electronics at this university. His research interest is in analog electronics and in electronic pictorial techniques. He is the author of numerous papers and holds several patents in his field of interest. He is the author of ten books, among which is Analog Electronic Circuit Design, Englewood Cliffs, NJ: Prentice Hall, 1991.

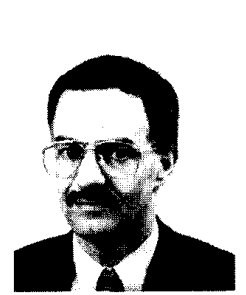

Arthur H. M. van Roermund was born in Delft, The Netherlands in 1951. He received the M.S. degree in electrical engineering in 1975 from the Delft University of Technology and the Ph.D. degree in Applied Sciences from the K. U. Leuven, Belgium, in 1987.

From 1975 to 1992 he was with the Philips Research Laboratories in Eindhoven. First he joined the Consumer Electronics Group, where he has been involved with the design and integration of analog circuits and systems, especially switched-capacitor circuits. In 1987 he joined the Visual Communications Group where he has been engaged in video architectures and digital video signal processing. From 1987 to 1990 he was project leader of the Video Signal Processor project and from 1990 to 1992 of a Multi-Window Television project. Since 1992 he has been a full professor at the Electrical Engineering Department of the Delft University of Technology. He heads the Electronics Laboratory, which is part of DIMES: the Delft Institute of Micro Electronics and Submicron technology. 